THE ASTROPHYSICAL JOURNAL, 565:1376-1380, 2002 February 1

(C) 2002. The American Astronomical Society. All rights reserved. Printed in U.S.A.

\title{
THE POTENTIAL FOR ATMOSPHERIC PATH LENGTH COMPENSATION IN STELLAR INTERFEROMETRY
}

\author{
C. H. TOWNES \\ Department of Physics, 366 Le Conte Hall 7300, University of California, Berkeley, Berkeley, CA 94720-7300 \\ Received 2001 August 17; accepted 2001 October 10
}

\begin{abstract}
Adaptive optics provides a method for improving telescope imaging affected by atmospheric seeing distortions, but the differences in path length fluctuations through the atmosphere to two or more separate telescopes continues to limit the quality of stellar interferometry, and unfortunately is not ameliorated by adaptive optics. Some corrections to such fluctuations can be made by atmospheric density measurements near the ground, particularly since in some cases a substantial fraction of path length fluctuations occur in the atmosphere within $20-30 \mathrm{~m}$ of ground level. It is shown that more extensive corrections can be made by measurements of backscattered lidar radiation sent along the direction of the telescopes' observation. It is proposed that Rayleigh or Raman backscattering be used to measure changes in atmospheric density or index of refraction. Rayleigh scattering by molecules provides more intensity than does Raman and can allow path length corrections over distances of a few hundred meters to somewhat more than a kilometer with a fractional accuracy as good as $10^{-6}$ of the total atmospheric delay. Such measurements can substantially assist stellar interferometry. Details of how this might be done, and of likely errors and difficulties, are discussed quantitatively.

Subject headings: instrumentation: adaptive optics - instrumentation: interferometers techniques: high angular resolution - techniques: interferometric
\end{abstract}

\section{INTRODUCTION}

The precision and sensitivity of spatial, or "stellar" interferometry done from the Earth's surface are usually limited by fluctuations in path lengths of stellar radiation through the atmosphere. Adaptive optics has recently been developed as a means of counteracting "seeing" fluctuations to improve images in a single telescope. For this, the wavefront of light arriving at a telescope is adjusted and made approximately planar over the telescope's aperture, thus producing an approximately diffraction limited image. However, this provides no correction for the "piston motions" or changes in relative path lengths to two separate telescopes which limit the quality of stellar interferometry. The relative fluctuations in atmospheric density along two or more parallel light paths from an object studied to the two or more separate telescopes used do not in themselves smear out images in a single telescope, but they rapidly change the relative phase of interfering beams and hence make difficult the measurements of maximum and minimum intensities in an interference pattern.

The atmospheric fluctuations in relative path lengths are usually more than one wavelength at optical or infrared wavelengths and make the interference between light collected by the two telescopes vary rapidly between maximum and minimum. As a result, interference fringes of the object to be observed may be badly smeared out. Experience with the magnitude and speed of fluctuations show wide variations, depending on atmospheric conditions, but indicate that path length corrections need to be made on a timescale as short as about $10^{-2} \mathrm{~s}$ for optical interference measurements, or about $0.2 \mathrm{~s}$ for interference of mid-IR $(10 \mu \mathrm{m})$ wavelengths. If there is a nearby star of sufficient intensity to give strong interference patterns and make possible rapid corrections in the optical paths, it can be used to compensate atmospheric path length fluctuations. But unfortunately, often there is no star close enough and of adequate intensity for rapid corrections to be made. As a result, for a star which is not bright, good interference fringes from its radiation may not be obtainable.

For possible improvement of interferometry, changes in path lengths through the atmosphere may be determined by measuring the amount of scattered light from a laser beam directed by a telescope along the same path over which astronomical radiation is received. If water droplets or aerosols are not abundant, the scattering is proportional to atmospheric molecular density, and a sufficiently precise measure of its amount can determine fractional changes in index of refraction or path length. However, the amount of scattering, the precision with which measurements can be made over appropriately short times, and the accuracy with which the scattering is proportional to the index of refraction must all be examined.

Rayleigh, Raman, and Mie scattering of pulsed laser beams, or lidar, have already been extensively used over the last few decades to examine densities of various atmospheric components. Lidar has provided useful measurements of gravity waves and temperature structure in the high atmosphere, and of molecular and dust components (Pal et al. 1996; Ansmann, Riebesell, \& Weitkamp 1990; Carswell et al. 1991; D'Altorio et al. 1993; Meriwether et al. 1994; Duck, Whiteway, \& Carswell 1998; Whiteway \& Carswell 1994; Whiteman, Melfi, \& Ferrare 1992). Doppler lidar has also measured velocities in the high atmosphere (Rothermel et al. 1991; Gal-Chen, Xu, \& Eberhard 1992). For a fixed atmospheric composition, the amount of Rayleigh scattering is proportional to the atmospheric density and hence can provide a measure of the index of refraction. Pulsed laser beams also allow determination of the location of the scattering and density measurement by timing of the scattered return signal. To determine densities of specific molecules, Raman scattering may be used. Mie scattering due to dust can be substantially greater than either Rayleigh or Raman scattering, but variation in dust density in the atmosphere both prevents its use for determination of atmo- 
spheric density and can badly confuse measurements of density by Rayleigh scattering. Actual use of scattering for an adequately precise measurement of optical or infrared path lengths clearly requires careful examination of the variations expected, the precision required, and methods which might obtain such precision.

\section{THE NATURE OF FLUCTUATIONS AND CORRECTIONS}

\section{TO BE MADE}

Density fluctuations in the atmosphere are usually approximated by the Kolmogorov model which, for two points separated by a distance $r$, predicts random relative fluctuations $\Delta \rho$ in density or $\Delta n$ in index of refraction proportional to $r^{5 / 6}$. The Taylor approximation assumes a constant wind moving the atmosphere, which implies path length variations in time proportional to $t^{5 / 6}$, or a power spectrum proportional to $v^{-5 / 3}$, where $v$ is the frequency of fluctuation. Although the Kolmogorov-Taylor model is only an approximation, it provides a useful model and reasonable estimates of the relative magnitude and timescales for fluctuations important to various wavelengths and various beam separations. Very near the Earth's surface or for large distances $r$ of separation, the model does not apply well.

A number of measurements of path length fluctuations through the atmosphere have been made; those associated with Berkeley's Infrared Spatial Interferometer (ISI) located at the Mount Wilson Observatory are examples. These show rough agreement with the power spectrum of fluctuations predicted by the Kolmogorov-Taylor approximation, but with a frequency variation of the power spectrum usually somewhat less that the predicted $v^{-5 / 3}$ (Bester et al. 1992). Amplitudes of fluctuations through the entire atmosphere are a few to a few tens of microns for reasonably good seeing. And under typical conditions at the Mount Wilson Observatory, about half of these fluctuations occur within 20-30 m height above the ground (Truehaft et al. 1995). The rapidity of path length fluctuations is highly variable depending on seeing conditions and wind speed. For most moderately good seeing conditions, the timescale for path length changes which are of the order $\lambda / 20$ is as short as about $0.01 \mathrm{~s}$ for visible light $(\lambda=500 \mathrm{~nm})$. For Kolmogorov-type fluctuations, this timescale varies as $\lambda^{6 / 5}$, so that at $10 \mu \mathrm{m}$ wavelength it is approximately $1 / 3 \mathrm{~s}$. Since it has been shown that one-half of the fluctuations occur within a few tens of meters of ground level, correction over a distance of only $20 \mathrm{~m}$ above ground level can be useful, and correction over the first $1000 \mathrm{~m}$ of path through the atmosphere may be very helpful. Hence, a goal for path length fluctuation correction might be to correct the $100-1000 \mathrm{~m}$ of lowest altitude in the path to a star with a precision of $\lambda / 40$ for mid-IR wavelengths. More ambitious goals would be to correct such distances with a precision adequate for optical wavelengths, or over still greater distances. Since the index of refraction of air is approximately $1+2.7 \times 10^{-4}$, the air itself lengthens the "path length" over a distance of $1000 \mathrm{~m}$ by $27 \mathrm{~cm}$. Hence, for a path length accuracy of $\lambda / 40$, where $\lambda=10 \mu \mathrm{m}$, changes in average air density over the path should be measured to a fractional precision of one part in $10^{6}$.

\section{MEASUREMENT OF DENSITY FLUCTUATIONS BY RAYLEIGH SCATTERING}

The cross section for Rayleigh backscattering by an individual air molecule is approximately $\sigma=5.45(550 /$ $\lambda)^{4} \times 10^{-28} \mathrm{~cm}^{2} \mathrm{sr}^{-1}$, where $\lambda$ is the wavelength in nanometers (Measures 1984). Hence, for light from an eximer $\mathrm{XeCl}$ laser with $\lambda=308 \mathrm{~nm}$, an STP atmosphere backscatters a fraction $1.49 \times 10^{-7}$ of the light per steradian per centimeter of air, or a total fraction of approximately $6.2 \times 10^{-7}$ of the light per centimeter. The backscatter is enough to provide a substantial scattered signal from a powerful lidar. The wavelength $308 \mathrm{~nm}$ is absorbed by ozone in the high atmosphere but is transmitted at lower altitudes except for the atmospheric scattering. And the total atmospheric scattering by molecules is small enough not to weaken the laser beam by more than about $50 \%$ as it traverses an entire atmosphere of $8 \mathrm{~km}$ at NTP density. This assumes there is no fog or dust to produce additional scattering and attenuation. In fact, scattering by particles is commonly intense enough to confuse any measurements of molecular density by total Rayleigh scattering. However, for a first analysis aerosol scattering will be ignored, and avoidance of aerosol scattering will be discussed later. An NTP atmosphere will also be assumed for the present discussion. At altitudes substantially above sea level, which is typical of observatories, the lower air density decreases scattering but also makes the precision needed less, which gives a modest net advantage.

Consider use of a $\mathrm{XeCl}$ laser of 50 watts average power and $308 \mathrm{~nm}$ wavelength pointed directly along a telescope's line of sight and pulsed 100 times per second with pulses $10^{-8} \mathrm{~s}$ long. This is similar to the laser used by Duck (1999) for stratospheric measurements and similar to some lasers presently used for the artificial stars of adaptive optics. Backscattered light from the short pulse, $3 \mathrm{~m}$ in length, allows location of the scattering to a precision of a few meters if that information is needed. Each pulse contains $7.8 \times 10^{17}$ photons and the backscatter per pulse from $3 \mathrm{~m}$ of STP air would be $3.5 \times 10^{13}$ photons $\mathrm{sr}^{-1}$. If backscattered light is collected by 65 inch diameter optics (such as that of the infrared spatial interferometer on Mount Wilson, cf. Hale et al. 2000) from a distance of $1000 \mathrm{~m}$, the number of photons collected from each $3 \mathrm{~m}$ pulse is $3.5 \times 10^{13} \times \pi / 4(1.65 / 1000)^{2}$, or $7.5 \times 10^{7}$. For measurement on a timescale of $0.3 \mathrm{~s}$, which is adequately fast under reasonable good conditions for atmospheric fluctuations affecting wavelengths as long as $10 \mu \mathrm{m}, 2.2 \times 10^{9}$ photons would be collected. This allows in principle a precision of one part in $4.7 \times 10^{4}$. Since the path length delay is 0.087 $\mathrm{cm}$ in $3 \mathrm{~m}$ of air, this gives an uncertainty of $1.9 \times 10^{-6} \mathrm{~cm}$ or $1.9 \times 10^{-3}$ wavelengths for $10 \mu \mathrm{m}$ radiation. Path length positions closer to the telescope receiving the radiation provide larger solid angles and hence more photons, so the precision could be greater, but for evaluation of air density over varying distances the number of photons would need to be normalized to allow for changes in intensity as a function of distance. If distances are measured by timing pulses and normalization is done to allow for the stronger signals at closer distances, which would also produce smaller fractional errors, then the total error over $1000 \mathrm{~m}$, summing the $3 \mathrm{~m}$ units, would be $2.0 \times 10^{-2} \lambda$, just slightly better than the goal of $\lambda / 40$.

The above calculation of course assumes perfect detection, precise measurement of detector response, and accurate calibration to provide correct compensation for path length fluctuations. It also assumes no scattering due to dust (or a constant fractional amount) and an atmosphere 
of constant composition (e.g., no variation in water content). High precision measurement of any atmospheric change is needed, but since the total fluctuations in path length which are rapid and need correction are normally only a few wavelengths, calibration of the overall scattering with an accuracy of only one part in a few hundred would be adequate and should not be difficult. Quantum efficiency of detection cannot be perfect but should be as large as $50 \%$, and this imperfect efficiency could be compensated by a factor of 2 increase in laser power. Dark current in the detector is not likely to be a problem since the photon flux would be large. However, good quantum efficiency and elimination of any short-term fluctuations in responses of associated circuitry would be important. Any fluorescence produced in the atmosphere can be eliminated by detecting light only in a narrow wavelength band at the laser frequency. Most problematic is scattering by atmospheric dust, which will be discussed later. But atmospheric dust could also cause difficulties by attenuating the transmitted radiation, thus producing some inaccuracies in the measurement. Even the scattering by atmospheric molecules would reduce the radiation intensity received from a round trip of light to a distance of $1000 \mathrm{~m}$ by about $12 \%$. This could require some compensation or might be a reason for using somewhat longer wavelengths which would have less scattering. However, it only represents an uncertainty in the calibration of any atmospheric change, and if such changes are no more than about $10 \mu \mathrm{m}$ due to atmospheric fluctuations at the higher altitudes, the $12 \%$ error would not be very serious.

Another problem, probably a minor one, is the possibility of variation in atmospheric composition, particularly in water vapor content. Water molecules scatter light and contribute to the atmosphere's index of refraction just as do the molecules $\mathrm{N}_{2}$ and $\mathrm{O}_{2}$ of dry air. However, the ratio of scattering to index of refraction contributions are not the same for water and dry air. Rayleigh scattering is proportional to $(n-1)^{2}$, where $n$ is the index of refraction of a gas at a standard molecular density; however, path length effects of the gas are proportional to $n-1$. Furthermore, $n-1$ is different for a given pressure of $\mathrm{H}_{2} \mathrm{O}$ and one of dry air at the proposed laser wavelength of $308 \mathrm{~nm}$, although essentially the same at $10 \mu \mathrm{m}$ wavelength. If an atmosphere at standard constant density changes its $\mathrm{H}_{2} \mathrm{O}$ content by 1 $\mathrm{mm}$, and its density change is judged by the amount of Rayleigh scattering, it can be estimated that the index of refraction at $10 \mu \mathrm{m}$ wavelength will be misjudged by approximately $8 \times 10^{-8}$. This would produce an error in assumed path length of $1 \mu \mathrm{m}$, or $\lambda / 10$ for $\lambda=10 \mu \mathrm{m}$, in a distance of $12.5 \mathrm{~m}$. It is not clear how rapidly humidity typically varies over short distances in a normal atmosphere, but changes over short distances, such as those between telescopes, which are as large as $1 \mathrm{~mm}$ of pressure seems unlikely under reasonably good conditions.

A technical problem which has so far been ignored is appropriate focussing of backscattered light at distances varying from $1000 \mathrm{~m}$ to perhaps only $10 \mathrm{~m}$ from the photon-collecting surface of the telescope. But it is in fact convenient and of some advantage to make no such variation in focus because a focus only at the longest distance to be used can ensure that the total backscatter received by a suitable detector is constant per unit path length, independent of distance to the scattering location. An aperture may, for example, be in front of the detector which admits only light focused by the receiving telescope within a solid angle subtended by the transmitted parallel beam at the maximum distance of $1000 \mathrm{~m}$. Since the solid angle received is constant, this reduces the fraction of the transmitted beam observed by the factor $(D / 1000)^{2}$, where $D$, the distance to the scattered light, is smaller than $1000 \mathrm{~m}$. Hence, the amount of light received per unit distance is constant out to $1000 \mathrm{~m}$, or at whatever distance the entire beam diameter is received. The ISI telescope's focal length of $5 \mathrm{~m}$ and diameter of $1.65 \mathrm{~m}$ requires an aperture at the detector of $0.82 \mathrm{~cm}$ to receive backscatter from the entire beam diameter at $1000 \mathrm{~m}$. This arrangement obviates any need for determining distances to the scattered radiation, or for renormalization of intensities received. It does, however, weaken signals from distances shorter than $1000 \mathrm{~m}$ and make probable errors per unit length constant over the path rather than improving at the shorter distances. As shown above, the theoretically allowed precision in determining path length due to $3 \mathrm{~m}$ of air at $1000 \mathrm{~m}$, using the laser power and collecting areas given as an example, is $2.0 \times 10^{-3}$ wavelengths at $10 \mu \mathrm{m}$. For the entire $1000 \mathrm{~m}$ in distance it would then be $(1000 / 3)^{1 / 2} \times 2.0 \times 10^{-3}$, or $3.7 \times 10^{-2}$ wavelengths. This is only a factor of 1.5 poorer than the goal suggested, and increased laser power could improve it. Although such an arrangement does not in principle provide quite as much precision as would the full use of all scattered light at distances shorter than $1000 \mathrm{~m}$ which was discussed earlier, it is substantially simpler and probably would in actual practice allow close to the same precision. Furthermore, since timing of returning pulses would not be necessary, it would be natural to use all backscattered light received by the detector for path length corrections, and this would include some light scattered from distances larger than $1000 \mathrm{~m}$, providing partial corrections for somewhat larger distances.

Another technical problem which would need to be dealt with is the effect of the proposed intense laser pulses on detection of stellar radiation for the interference process. Since the laser wavelength would be different from that where interference is being measured (in the example given $308 \mathrm{~nm}$ vs. $10 \mu \mathrm{m}$ ), the interferometer detectors can be isolated by dichroic beam splitters and filters. If reasonably fast detectors are used, they might also be isolated from pulsed laser light by pulsed control of bias voltages without appreciable loss of signal integration time.

The example discussed here involves path length corrections adequate for substantial improvement of interferometry in the mid IR region. For optical wavelengths some corrections could be made by similar techniques, but they would be more limited. The shorter wavelengths would require an order of magnitude more precision in path length measurement, and the timescale of fluctuations would require measurements on a timescale at least an order of magnitude shorter. However, measurements could be made to eliminate fluctuations closer to the ground than $1000 \mathrm{~m}$ or to give approximate corrections over larger distances. Such approximate corrections may simplify the mechanical tracking of path length changes of the type which is normally done for stellar interferometry at optical wavelengths.

The most substantial uncertainty in the path length measurement procedure outlined above is the likely presence of dust or other aerosols in the line of sight which could backscatter more light than the molecules. If the aerosol concentration were constant throughout the path being measured, 
this would not cause a problem, because any fluctuations would still represent fluctuations in air density. However, variations in aerosol concentration must be expected. And in many cases at low altitudes, aerosol scattering is substantially larger than Rayleigh scattering from molecules and is likely to make useful precision in density fluctuations unobtainable. The use of short wavelengths tends to minimize the ratio of Mie to Rayleigh scattering, and there may be some astronomical observing sites at relatively high altitudes where aerosol concentration would not be a severe problem for the type of measurement outlined above. However, measurements of a dry atmosphere at a kilometer altitude and higher have shown aerosols, presumably manmade, abundant enough to produce Mie scattering within an order of magnitude as large as that of the air molecules (Müller et al. 1998). And fluctuations in relative aerosol density would cause problems with measurement of air density variations. Aerosol fluctuations would clearly need to be examined carefully at any particular site if measurements of the type discussed above are to be used.

\section{MEASUREMENT OF DENSITY FLUCTUATIONS BY RAMAN SCATTERING}

An alternative to Rayleigh scattering as a measure of density fluctuations is Raman scattering, since a specific Raman frequency identifies a particular molecular component and cannot be produced by Mie scattering from aerosols. Its possible use will hence be discussed. The Raman backscatter cross section from a nitrogen molecule for incident light of the wavelength $308 \mathrm{~nm}$ considered above is approximately $4 \times 10^{-30} \mathrm{~cm}^{2} \mathrm{sr}^{-1}$, making $\mathrm{N}_{2}$ Raman scattering in the atmosphere roughly 1000 times smaller than the total Rayleigh scattering. This would decrease precision by a factor of about 30 for the same laser intensity and collection techniques discussed above. Such a decrease, with a resulting precision in measurement of path length changes of only $\frac{3}{4} \lambda$ for $\lambda=10 \mu \mathrm{m}$, would not by itself provide good interferometric measurements, so other modifications in parameters need to be considered.

The general arrangement discussed above for collection of photons of the same number per unit path length regardless of the distance appears very convenient and does not lose precision by any large amount, so it will be assumed to always apply while other parameters are changed. For this case, the number of photons which must be collected is proportional to the square of the total distance $L$ over which measurements are to be made, since the fractional precision must be proportional to $1 / L$ and the randomness of scattering produces fluctuations in numbers of photons proportional to the square root of the number. The number collected per united length is proportional to $1 / L^{2}$, where $L$ is the maximum distance of the measurement. And the total number of photons collected is

$$
n=\frac{a \sigma P D^{2} t}{L},
$$

where $a$ is a proportionality constant, $\sigma$ is the scattering cross section per molecule, $P$ is the laser power, $L$ is the total path length over which collection occurs, $D$ is the diameter of the telescope aperture receiving the photons, and $t$ is the time over which photons are collected in order to measure path length fluctuations.

Since the number of photons which must be collected to provide a given precision is proportional to $L^{2}$, to use Raman scattering from $\mathrm{N}_{2}$ molecules over a distance $L_{1}$ rather than Rayleigh scattering from all molecules over a distance $L$ requires

$$
\frac{n_{1}}{L_{1}^{2}}=\frac{n}{L^{2}}
$$

or

$$
\frac{\sigma_{1} P_{1} D^{2} t_{1}}{L_{1}^{3}}=\frac{\sigma P D^{2} t}{L^{3}},
$$

where the quantities with subscript 1 apply to the Raman scattering case and the others to Rayleigh scattering. For the same power, telescope diameter, and time, $L_{1}^{3}=L^{3} \sigma_{1} / \sigma$, or $L_{1}=(1 / 10) L$ since $\sigma_{1} \approx 10^{-3} \sigma$.

If path length fluctuations can be measured only over the first $100 \mathrm{~m}$ of path by Raman scattering instead of $1000 \mathrm{~m}$, is this large enough to be useful? As noted above, Truehaft et al. (1995) have shown that under normal observing conditions on Mount Wilson, approximately $50 \%$ of the path length fluctuations occur in the first $20-30 \mathrm{~m}$ along the line of sight of an observing telescope. While conditions can be quite variable, and it is not clear how much of the total path length fluctuations occur in the next $75 \mathrm{~m}$ beyond the first 25 , it appears that path length corrections over a distance in the atmosphere even as short as $100 \mathrm{~m}$, which appears practical with Raman scattering, may be quite useful.

To what extent can Raman scattering be extended beyond $100 \mathrm{~m}$ ? Since from the above expressions,

$$
L_{1}=L\left(\frac{\sigma_{1} P_{1} D_{1}^{2} t_{1}}{\sigma P D^{2} t}\right)^{1 / 3},
$$

one can consider increasing power, perhaps by a factor as large as 10, and using a large subsidiary "light bucket" collector with diameter $D_{1}$ of $5 \mathrm{~m}$ instead of the value 1.65 $\mathrm{m}$ for $D$. This would increase $L_{1}$ to $450 \mathrm{~m}$, which should provide useful path length corrections.

Although the ratio of $\mathrm{N}_{2}$ to $\mathrm{O}_{2}$ in the atmosphere probably does not vary appreciably over a short time, the amount of $\mathrm{H}_{2} \mathrm{O}$ may vary, which means that measurement of $\mathrm{N}_{2}$ densities alone may not allow a determination of variations in optical path lengths to sufficient accuracy. The probability of short-term fluctuations in water vapor content is not well known and would need to be measured in order to determine how seriously this affects path lengths. If in fact it does change path lengths in a troublesome way, the water vapor density in the optical path would need to be measured as well as that of $\mathrm{N}_{2}$. This could be done by Raman scattering of the laser radiation by $\mathrm{H}_{2} \mathrm{O}$. The Raman scattering cross section of $\mathrm{H}_{2} \mathrm{O}$ is approximately $2 \times 10^{-29} \mathrm{~cm}^{2} \mathrm{sr}^{-1}$ at a wavelength of $308 \mathrm{~nm}$, which is 5 times larger than that of $\mathrm{N}_{2}$. Detection of the separate Raman spectra of $\mathrm{N}_{2}$ and $\mathrm{H}_{2} \mathrm{O}$ would be needed, but would not add much complexity to a system designed to isolate the Raman spectrum of $\mathrm{N}_{2}$ alone.

\section{RESOLUTION OF AEROSOL FROM MOLECULAR SCATTERING - PROBABLY THE OPTIMAL APPROACH}

If aerosol scattering is large enough to interfere with accurate measurement of atmospheric density by Rayleigh backscattering, as is probably commonly the case, it may be spectrally separated from molecular scattering. This is an 
alternative to the use of Raman scattering, which will provide substantially more scattered intensity and precision than the Raman scattering possibility.

Backscattered light from air molecules will be Doppler shifted due to kinetic motion of the molecules and spread out over a fractional spectral range of approximately

$$
\frac{2}{c} \sqrt{\frac{2 k T}{m}},
$$

where $m$ is the molecular mass, $T$ is the air temperature, and $k$ and $c$ are the Boltzmann constant and velocity of light, respectively. For a wavelength of $308 \mathrm{~nm}$, this corresponds to a spread of $\pm 0.09 \mathrm{~cm}^{-1}$ for $\mathrm{N}_{2}$ molecules at a temperature of approximately $300 \mathrm{~K}$. The frequency spread due to thermal motions of aerosols or the wind velocity would be no larger than about $0.001 \mathrm{~cm}^{-1}$. Hence, high spectral resolution which eliminates the Mie scattering by aerosols could allow approximately $90 \%$ of the Rayleigh scattering by molecules to be used for air density measurements. This would provide more photons by a factor of about 1000 than would the use of Raman scattering. It could thus provide more precision in density measurements over large distances than the Raman scattering technique discussed above, though it could not make separate measurements of both $\mathrm{N}_{2}$ and $\mathrm{H}_{2} \mathrm{O}$ as could the use of Raman scattering.

For the spectral resolution needed to separate molecular from aerosol scattering, the laser used must have a spectrum as narrow as a few hundredths of a wavenumber. Pulses of $10^{-8} \mathrm{~s}$ would imply widths of about $0.001 \mathrm{~cm}^{-1}$ and hence would not produce a troublesomely wide spectrum. Spectral separation of aerosol and of molecular scattering has already been demonstrated. For example, Piironen \& Eloranta (1994) have used a tunable Nd:Yg laser and absorption of its radiation by iodine vapor to remove the aerosol backscatter. Precision of these earlier experiments was not enough to measure atmospheric densities adequately for stellar interferometry, but the techniques used can be applied for this purpose.

Removal of the narrow spectrum of light due to Mie scattering by aerosols should allow the precision and the measurement of path lengths to be essentially similar to those discussed in the earlier section on Rayleigh scattering. It would not allow separate corrections for water vapor content as would measurement of Raman scattering, but path length problems due to rapid variation in water vapor density would probably not be large. Hence, this approach appears to be the more promising way of obtaining substantial path length fluctuation measurement and correction.

\section{SUMMARY}

Although correction of path length fluctuations through the complete atmosphere probably cannot be made by measuring backscattered light from a laser beam probe to the accuracy needed for stellar interferometry, it appears that measurements of path lengths can be made over large enough distances to improve such interferometry. The present proposals consider, in particular, corrections over path lengths of $100 \mathrm{~m}$ to $1000 \mathrm{~m}$ from the ground, where a large fraction of the total path length variations through the atmosphere have been shown to occur at least under some observing conditions. The precision achievable appears adequate to substantially improve interferometry in the mid-IR (10 $\mu \mathrm{m}$ wavelength) region. At optical wavelengths, the precision required is much more demanding, and the distances over which good corrections could be made can be expected to be at least on order of magnitude less that those for the mid-IR region. Nevertheless, some useful corrections might be achieved for wavelengths as short as visible light.

Further study of the nature of path length variations would be appropriate before systems for their correction are completely designed or planned. This might include better knowledge of the relative magnitude and variations of aerosol to molecular scattering near the ground and the relative importance of water vapor concentration variations compared to atmospheric density fluctuations. And further knowledge of the path length fluctuations within 100-1000 $\mathrm{m}$ of the ground compared to those in the higher atmospheres would be important. Although uncertainties remain as to the extent of corrections which can reasonably be made, it does appear practical to make partial corrections that can give significant improvements to the quality of stellar interferometry from ground observatories.

This work has been sponsored by the Army Research Office and by the National Science Foundation. I want to also express my special appreciation to John W. Meriwether of Clemson University and Thomas Duck of the Haystack Observatory, Massachusetts Institute of Technology, whose lidar work on the upper atmosphere suggested to me the application of lidar to interferometry, and who helped me with much background information. I also appreciate helpful comments of Professors Edwin Eloranta of the University of Wisconsin and Raymond Measures of the University of Toronto.

\section{REFERENCES}

Ansmann, A., Riebesell, M., \& Weitkamp, C. 1990, Opt. Lett., 15, 746

Bester, M., Danchi, W. C., Degiacomi, C. G., Greenhill, L. J., \& Townes, C. H. 1992, ApJ, 392, 357

Carswell, A. I., Pal, P. R., Streinbrecht, W., Whiteway, J. A., Ulitsky, V., \& Wang, T. Y. 1991, Canadian J. Phys., 69, 1076

D’Altorio, S., Masei, F., Rizi, V., Visconti, G., \& Verdecchia, M. 1993, Geophys. Res. Lett., 20, 2869

Duck, T. J., Whiteway, J. A., \& Carswell, A. J. 1998, Geophys. Res. Lett., 25,2813

Duck, T. J. 1999, Ph.D. thesis, York Univ., Canada

Gal-Chen, T. M., Xu, M., \& Eberhard, W. L. 1992, J. Geophys. Res., 97, 18409

Hale, D. D. S., et al. 2000, ApJ, 537, 998

Measures, R. M. 1984, Laser Remote Sensing-Fundamentals and Applications (New York: Wiley)
Meriwether, J. W., Dao, P. D., McNutt, R. J., Klementi, W., Moskowitz, W., \& Davidson, W. 1994, J. Geophys. Res., 99, 16973

Müller, D., Wandinger, U., Althausen, D., Mattis, I., \& Ansmann, A. 1998, Appl. Opt., 37, 2260

Pal, S. R., Carswell, A. J., Bird, J., Donavan, D., Duck, T., \& Whiteway, J. 1996, Proc. SPIE, 2833, 28

Piironen, P., \& Eloranta, E. W. 1994, Opt. Lett., 19, 234

Rothermel, J., Bowdle, D. A., Vaughn, J. M., \& Woodfield, A. A. 1991, J. Geophys. Res., 96, 5293

Truehaft, R. N., Lowe, S. T., Bester, M., Danchi, W. C., \& Townes, C. H. 1995, ApJ, 453, 522

Whiteman, D. N., Melfi, S. H., \& Ferrare, R. A. 1992, Appl. Opt., 31, 3058

Whiteway, J. A., \& Carswell, A. J. 1994, J. Atmos. Sci., 51, 3122 